\title{
Viabilidad del uso de cenizas de vinaza como fertilizante para forrajeras
}

\author{
Viabilidade do uso de cinzas de vinhaça como fertilizantes \\ para forrageiras
}

Viability of vinasse ashes use as fertilizer to forage plants

Luciano Ricardo de Oliveira ${ }^{1}$ Danila Fernanda Rodrigues Frias ${ }^{2}$ André da Cruz França Lema ${ }^{3}$ Roberto Andreani Júnior ${ }^{4}$

${ }^{1}$ Estudiante de Agronomía en la Universidade Brasil. E-mail: luciano.ricardo1.Ir@gmail.com, ORCID: http://orcid.org/0000-0002-8084-8014

${ }^{2}$ Profesora del Programa de Maestría en Ciencias Ambientales en la Universidade Brasil. E-mail: danila.frias@universidadebrasil.edu.br, ORCID: http://orcid.org/0000-0001-8621-3338

${ }^{3}$ Profesor en la Universidade Brasil y en el Instituto Federal do Sul de Minas. E-mail: andrelema@uol.com.br, ORCID: http://orcid.org/0000-0001-8848-1862

${ }^{4}$ Profesor del Programa de Maestría en Ciencias Ambientales en la Universidade Brasil. E-mail: robertoandreani@uol.com.br, ORCID: http://orcid.org/0000-0002-0290-3356 
Resumen: Fue evaluada la utilización de cenizas de vinaza como fertilizante para forrajeras. La vinaza fue llevada a mufla a 600ㄷ por 90 minutos para producción de las cenizas. Se sembró la forrajera Urochloa brizantha - cv. Marandu, en bolsas plásticas de polietileno que se mantuvieron en casa de vegetación. Los tratamientos utilizados fueron la aplicación de ceniza de vinaza en cantidades equivalentes a $0,100,250,500,1000 \mathrm{~kg} / \mathrm{ha}$. Las plantas fueron cortadas con 30 días y 60 días para evaluar la producción de materia seca (MS) y materia verde (MV). En el suelo enriquecido, se retiró una muestra para realizar análisis. Hubo corrección de pH del suelo y aumento de los macrominerales fósforo, potasio, calcio y magnesio. El tratamiento con $100 \mathrm{~kg} /$ ha se mostró más eficaz e elevó en promedio el 18,5\% la producción de MV y el $24 \%$ la producción de MS de la forrajera estudiada. Se concluye que el uso de cenizas de vinaza es una potencial fuente de minerales para forrajeras.

Palavras-chave: reservación del medio ambiente; producción sucroalcoholera; sostenibilidad; Urochloa brizantha - cv. Marandu.

Resumo: Avaliou-se a utilização de cinzas de vinhaça como fertilizante para forrageiras. A vinhaça foi levada a mufla a 600 ㄷ por 90 minutos para produção das cinzas. Semeou-se a forrageira Urochloa brizantha - cv. Marandu, em sacos plásticos de polietileno que foram mantidos em casa de vegetação. Os tratamentos utilizados foram aplicação de cinza de vinhaça em quantidades equivalentes a $0,100,250,500,1000 \mathrm{~kg} / \mathrm{ha}$. As plantas foram cortadas com 30 dias e 60 dias para avaliar a produção de matéria seca (MS) e matéria verde (MV). Do solo enriquecido, foi retirada uma amostra para realização de análise. Houve correção de pH do solo e aumento dos macrominerais fósforo, potássio, cálcio e magnésio. 0 tratamento com $100 \mathrm{~kg} / \mathrm{ha}$ mostrou-se mais eficaz e elevou em média $18,5 \%$ a produção de MV e $24 \%$ a produção de MS da forrageira estudada. Conclui-se que o uso de cinzas de vinhaça é uma potencial fonte de minerais para forrageiras.

Keywords: preservação do meio ambiente; produção sucroalcoleira; sustentabilidade; Urochloa brizantha - cv. Marandu.

\begin{abstract}
We evaluated the use of vinasse ashes as fertilizer to forage plants. We put the vinasse to the muffle furnace at $600^{\circ} \mathrm{C}$ for 90 minutes to the production of ashes. It was sowed the forage plant Urochloa brizantha - cv Marandu, in polyethylene plastic bags that were kept in a greenhouse. The treatments used were application of vinasse ashes equivalent to $0,100,250,500,1000 \mathrm{~kg} / \mathrm{ha}$. We cut the plants at 30 days and 60 days in order to evaluate the production of dry matter (DM) and green matter (GM). From the soil enriched with, we took a sample to analyze. There was a correction of $\mathrm{pH}$ of the soil and an increase of the macrominerals phosphorus, potassium, calcium, and magnesium. The treatment with $100 \mathrm{~kg} / \mathrm{ha}$ has proved to be more effective and increase on average of $18.5 \%$ the production of GM and of $24 \%$ the production of DM of the forage plant studied. We concluded that the use of vinasse ashes is a potential source of minerals to the forage plants.
\end{abstract}

Palabras clave: environmental preservation; sugarcane alcohol production; sustainability; Urochloa brizantha - cv. Marandu. 


\section{INTRODUCCIÓN}

Actualmente la explotación de la caña de azúcar es una de las actividades productivas en crecimiento en Brasil, y debido a la ampliación de la producción sucroalcoholera y la disposición inadecuada de los residuos sólidos generados por este seguimiento, graves problemas ambientales relacionados con la contaminación se están produciendo en el país.

La vinaza es un efluente producido por la industria sucroalcoholera siendo considerado el principal subproducto del proceso de fabricación del etanol y de la cachaça, pues para cada litro de etanol obtenido se producen trece litros de vinaza.

Una de las utilizaciones actuales de la vinaza es la fertirrigación de la caña de azúcar, pero la aplicación excesiva y continua de este efluente con alta carga orgánica y de minerales como el potasio, calcio, magnesio, además de nitratos y azufre, se infiltra en el suelo, pudiendo contaminar las aguas subterráneas y causar la salinización de los acuíferos. Además, la viña tiene un poder contaminante cerca de cien veces mayor que el desagüe doméstico debido a la alta carga orgánica que posee.

El residuo citado, aun siendo muy discutido y abordado como factor perjudicial a los nichos ecológicos, aún no tiene destino adecuado, y aunque es blanco de polémica, ese efluente posee una vasta riqueza mineral escondida, sobre todo de K, Ca, Mg, S y N. Así, la utilización de este subproducto para el desarrollo sostenible debe ser una práctica adoptada en la gestión de los residuos de las industrias sucroalcoholeras.

Ante el crecimiento del sector sucroalcoholero en Brasil, y de la necesidad de gestión de este residuo cuya disposición directa en el suelo representa una solución ambientalmente desventajosa, la utilización de su procesamiento y de éste como fertilizante viene siendo estudiada.

Una alternativa al uso de la vinaza sería su transformación en cenizas, ya que, la utilización de cenizas en la agricultura es considerada por muchos como ambientalmente correcta, debido a la utilización de todos los residuos en el proceso de producción. Los suelos de áreas cultivables comúnmente sufren desequilibrio en su fertilidad, ocasionando el surgimiento de deficiencias nutricionales derivadas del cultivo intenso y continuo. Así, la corrección de la 
fertilidad del suelo es comúnmente realizada con la utilización de abonos químicos, los cuales se obtienen a partir de fuentes potencialmente agotadoras.

La utilización de la ceniza vegetal en Urochloa brizantha para corrección y fertilidad del suelo es una óptima opción para suelos de baja fertilidad como los tropicales de cerrado, por ejemplo. Además de la viabilidad en la producción de la forrajera, es una forma de descarte de este residuo en el ambiente, ya que es producido en grandes cantidades por las industrias y calderas en general. Por ello, el objetivo de este trabajo fue evaluar la utilización de cenizas de vinaza como fertilizantes y potencial fuente de minerales para forrajeras para atenuar su utilización en la fertirrigación de la caña de azúcar disminuyendo el impacto ambiental producido, además de la posibilidad de agregar valor al subproducto mencionado.

\section{MATERIAL Y MÉTODOS}

El experimento fue realizado en las dependencias de la Universidad Brasil- Campus Fernandópolis, SP.

La vinaza fue obtenida de una usina sucroalcoholera de la región Noroeste del Estado de São Paulo.

Para la producción de las cenizas, la vinaza fue colocada en invernadero a 100 ㄷ por 6 horas para evaporación de parte del agua. En seguida fue Ilevada la mufla y expuesta a 600 ㄷ C por 90 minutos para obtener las cenizas.

La planta forrajera utilizada fue la Urochloa brizantha- cv. Marandu, cultivada en condiciones de casa de vegetación, en bolsas plásticas de polietileno, visando mayor control de las condiciones ambientales, durante el período experimental.

Antes de la siembra, se realizó la prueba de germinación de las semillas. Para la quiebra del entumecimiento las semillas quedaron sumergidas en solución de nitrato de potasio al 2\% por 24 horas. En seguida 100 semillas fueron acondicionadas en papel gerbox húmedo, divididas en cuatro placas de Petri, siendo evaluadas diariamente.

También se realizó el análisis del suelo que se utilizó para la siembra, sin adición de cenizas de vinaza y después de la adición de las cenizas con una equivalencia de $1000 \mathrm{~kg} / \mathrm{ha}$. 
Después de la prueba de germinación y análisis del suelo se realizó el plantío. Los tratamientos consistieron en la aplicación de ceniza de vinaza en cantidades equivalentes a 0 (testigo), 100, 250, 500, $1000 \mathrm{~kg} / \mathrm{ha}$, sin fertilización química de plantío. Se utilizaron 10 repeticiones por tratamiento, siendo el tratamiento 1 (T1) testigo, tratamiento 2 (T2) $100 \mathrm{~kg} / \mathrm{ha}$, tratamiento 3 (T3) $250 \mathrm{~kg} / \mathrm{ha}$, tratamiento 4 (T4) $500 \mathrm{~kg} / \mathrm{ha}$ y tratamiento 5 (T5) $1000 \mathrm{~kg} / \mathrm{ha}$.

Las plantas fueron asignadas en casa de vegetación de forma casualizada. Después de 30 días de la emergencia de las plántulas, fue realizado el primer corte simulando el pastoreo. El material recolectado fue pesado para la obtención de materia verde (MV). En seguida fue acondicionado en bolsas de papel y colocado en invernadero a $56^{\circ} \mathrm{C}$ con circulación de aire forzada por 48 horas. Después de este período el material fue pesado para la obtención de materia seca (MS). Este procedimiento se repitió después de 30 días del primer corte, es decir, después de 60 días de la emergencia de las plántulas.

Pasados 90 días de la emergencia de las plántulas, las mismas fueron recogidas íntegramente. Se realizó el pesaje de la parte aérea para la obtención de materia verde, y el pesaje y medición del sistema radicular. A continuación, la parte aérea fue acondicionada en bolsas de papel y colocada en un invernadero a 56으 con circulación de aire forzada durante 48 horas, para obtener la materia seca.

Los datos obtenidos se evaluaron mediante la prueba de Duncan con Alpha 0,1.

\section{RESULTADOS Y DISCUSIÓN}

Para el inicio del estudio, el suelo utilizado para la siembra de la forrajera fue sometido a la realización de análisis. El resultado obtenido se describe en la Tabla 1. 
Tabla 1 - Análisis del suelo utilizado para plantío de la forrajera Urochloa brizantha cv. Marandu, antes de la incorporación de las cenizas de viña

\begin{tabular}{ccc}
\hline Determinaciones & Unidad de medida & Resultado \\
\hline Matéria orgánica & $\mathrm{g} / \mathrm{dm}^{-3}$ & 17 \\
Fosforo resina & $\mathrm{mg} / \mathrm{dm}^{-3}$ & 13 \\
$\mathrm{pH}$ & & 5,5 \\
Potasio & $\mathrm{Mmol}_{\mathrm{C}} / \mathrm{dm}^{-3}$ & 1,6 \\
Calcio & $\mathrm{Mmol}_{\mathrm{C}} / \mathrm{dm}^{-3}$ & 15 \\
Magnesio & $\mathrm{Mmol}_{\mathrm{C}} / \mathrm{dm}^{-3}$ & 6 \\
Acidez potencial & $\mathrm{Mmol}_{\mathrm{C}} / \mathrm{dm}^{-3}$ & 22 \\
Suma de bases & $\mathrm{Mmol}_{\mathrm{C}} / \mathrm{dm}^{-3}$ & 22,6 \\
Cambio catiônica & $\mathrm{Mmol} / \mathrm{dm}^{-3}$ & 44,60 \\
Saturación por bases & $\%$ & 50,67 \\
\hline
\end{tabular}

Fuente: Autoría propia.

Se produjeron 150 gramos de cenizas con aproximadamente 30 litros de vinaza. Estas cenizas se incorporaron al suelo en cantidades equivalentes a $100,250,500$ y $1000 \mathrm{~kg} / \mathrm{ha}$. En el suelo enriquecido con la equivalencia de $1000 \mathrm{~kg} /$ ha, se retiró una muestra para realizar análisis. El resultado se describe en la Tabla 2.

Tabla 2 - Análisis del suelo utilizado para plantío de la forrajera Urochloa brizantha cv. Marandu, después de la incorporación de las cenizas de viña

\begin{tabular}{ccc}
\hline Determinaciones & Unidad de medida & Resultado \\
\hline Matéria orgánica & $\mathrm{g} / \mathrm{dm}^{-3}$ & 18 \\
Fosforo resina & $\mathrm{mg} / \mathrm{dm}^{-3}$ & 15 \\
$\mathrm{pH}$ & & 6,5 \\
Potasio & $\mathrm{Mmol}_{\mathrm{c}} / \mathrm{dm}^{-3}$ & 2,4 \\
Calcio & $\mathrm{Mmol}_{\mathrm{C}} / \mathrm{dm}^{-3}$ & 19 \\
Magnesio & $\mathrm{Mmol}_{\mathrm{C}} / \mathrm{dm}^{-3}$ & 7 \\
Acidez potencial & $\mathrm{Mmol}_{\mathrm{C}} / \mathrm{dm}^{-3}$ & 17 \\
Suma de bases & $\mathrm{Mmol}_{\mathrm{c}} / \mathrm{dm}^{-3}$ & 28,4 \\
Cambio catiônica & $\mathrm{Mmol}_{\mathrm{c}} / \mathrm{dm}^{-3}$ & 45,40 \\
Saturación por bases & $\%$ & 62,56 \\
\hline
\end{tabular}

Fuente: Autoría propia.

Se observó que después de la adición de las cenizas ocurrió alteración en las características del suelo aumentando los valores en todos los 
ítems evaluados. Se destaca la capacidad para corregir el pH, haciéndolo más cercano a la neutralidad, y el aumento de los macrominerales fósforo, potasio, calcio y magnesio.

La reacción de las dosis de las cenizas en el suelo en este trabajo proporciona que los carbonatos presentes en el residuo elevar el pH del suelo, así como ocurrió en experimento de Ferreira, Fageria y Didonet (2012).

El fósforo es un elemento importante que estimula la formación y el crecimiento radicular siendo responsable de los procesos energéticos en las plantas y está entre los nutrientes de mayor limitación en la productividad de éstas, aunque absorbido en menores cantidades que el potasio y el nitrógeno, lo que resulta en bajos en las hojas (MALAVOLTA, 1987; RAIJ, 1991). En su ausencia, se considera el macronutriente responsable de la baja productividad en los pastos en suelos de baja fertilidad y con acidez elevada, siendo el elemento más importante en la formación de pastos en el bioma cerrado (VILELA et al., 2007). En solución nutritiva, trabajando con el pasto marandu, Monteiro et al. (1995) observaron un aumento en la concentración de fósforo en las raíces de esta gramínea.

Los mismos autores en ensayo con fertilización química en pasto marandu encontraron una elevación en los niveles de potasio en la raíz de la forrajera, y esto debe haber ocurrido por las cantidades elevadas de este elemento en la ceniza vegetal utilizada en este experimento, lo que corrobora con nuestros resultados.

La presencia del calcio es fundamental para el crecimiento de los tejidos meristemáticos y funcionamiento de los ápices radiculares mejorando así, el crecimiento de las raíces de las plantas (DECHEN; NACHTIGALL, 2007). Como la mayoría de los suelos tropicales es pobre en calcio, el suministro de este elemento a través de las cenizas vegetales altera la proporción de este nutriente en las plantas regulando el metabolismo en las mismas (OLIVEIRA et al., 2005; COSTA et al., 2008).

El magnesio participa en la molécula de clorofila en el proceso fotosintético de las plantas, a pesar de su exigencia por las plantas ser modestas (RAIJ, 1991). Segundo Monteiro et al. (1995), la masa seca de la raíz del pasto marandu cultivado en solución nutritiva varía de 0,4 a 3,5 del tratamiento con el nutriente con relación al tratamiento sin magnesio. 
El suelo enriquecido con las cenizas y el control fueron colocados en bolsas plásticas de polietileno y distribuidos aleatoriamente en casa de vegetación. Después de la prueba de germinación apuntar el 79\%, se sembraron 5 semillas por saco con profundidad de $1,5 \mathrm{~cm}$ en distribución uniforme. Después de la realización de la plantación, en 7 días, las plantas emergieron y quedaron bajo seguimiento en casa de vegetación hasta llegar al punto del primer corte (30 días después de la emergencia). Los datos obtenidos en el primer corte están dispuestos en la Tabla 3.

Tabla 3 - Resultados obtenidos relacionados con la producción de materia verde (MV) y materia seca (MS), por tratamiento (T), por repetición (Rep), de la muestra recogida 30 días después de la emergencia de las plántulas

\begin{tabular}{|c|c|c|c|c|c|c|c|c|c|c|}
\hline \multirow{2}{*}{ Rep } & \multicolumn{2}{|c|}{ T1 } & \multicolumn{2}{|c|}{ T2 } & \multicolumn{2}{|c|}{ T3 } & \multicolumn{2}{|c|}{ T4 } & \multicolumn{2}{|c|}{ T5 } \\
\hline & MV (g) & MS (g) & $\mathrm{MV}(\mathrm{g})$ & MS (g) & MV (g) & MS (g) & MV (g) & MS (g) & MV (g) & MS (g) \\
\hline 1 & 2,05 & 0,3 & 1,44 & 0,26 & 3,13 & 0,52 & 2,88 & 0,49 & 3,56 & 0,58 \\
\hline 2 & 2,18 & 0,33 & 3,43 & 0,56 & 3,59 & 0,56 & 2,59 & 0,50 & 0,42 & 0,08 \\
\hline 3 & 0,67 & 0,15 & 0,89 & 0,16 & 3,69 & 0,62 & 3,32 & 0,53 & 3,86 & 0,59 \\
\hline 4 & 2,17 & 0,33 & 2,70 & 0,44 & 1,79 & 0,26 & 3,59 & 0,56 & 2,50 & 0,37 \\
\hline 5 & 3,22 & 0,50 & 1,25 & 0,2 & 1,68 & 0,25 & 1,59 & 0,30 & 4,35 & 0,70 \\
\hline 6 & 1,87 & 0,27 & 2,87 & 0,43 & 1,34 & 0,20 & 0,85 & 0,14 & 2,01 & 0,30 \\
\hline 7 & 1,88 & 0,32 & 2,99 & 0,46 & 0,45 & 0,08 & 1,39 & 0,20 & 2,32 & 0,32 \\
\hline 8 & 0,66 & 0,08 & 4,08 & 0,64 & 3,73 & 0,54 & 0,39 & 0,07 & 1,00 & 0,16 \\
\hline 9 & 4,14 & 0,72 & 3,78 & 0,59 & 2,29 & 0,33 & 3,45 & 0,56 & 1,29 & 0,23 \\
\hline 10 & 2,09 & 0,33 & 2,71 & 0,43 & 3,05 & 0,46 & 0,48 & 0,09 & 1,89 & 0,32 \\
\hline
\end{tabular}

Fuente: Autoría propia.

Después de 60 días de emergencia de las plántulas, se realizó el segundo corte, cuyos resultados se describen en la Tabla 4.

Tabla 4 - Los resultados obtenidos relacionados con la producción de materia verde (MV) y la materia seca (MS), por tratamiento (T), por repetición (Rep), de la muestra recogida 60 días después de la emergencia de las plántulas

\begin{tabular}{|c|c|c|c|c|c|c|c|c|c|c|}
\hline Rep & \multicolumn{2}{|c|}{ T1 } & \multicolumn{2}{|c|}{ T2 } & \multicolumn{2}{|c|}{ T3 } & \multicolumn{2}{|c|}{ T4 } & \multicolumn{2}{|c|}{ T5 } \\
\hline & $\mathrm{MV}(\mathrm{g})$ & MS (g) & $\mathrm{MV}(\mathrm{g})$ & MS (g) & MV (g) & MS (g) & $\mathrm{MV}(\mathrm{g})$ & MS (g) & MV (g) & $\mathrm{MS}(\mathrm{g})$ \\
\hline 1 & 2,63 & 0,45 & 6,57 & 1,30 & 1,43 & 0,21 & 7,79 & 1,72 & 2,86 & 0,44 \\
\hline 2 & 8,18 & 1,45 & 4,40 & 0,70 & 6,76 & 1,33 & 6,27 & 1,31 & 1,48 & 0,27 \\
\hline 3 & 6,38 & 1,20 & 8,60 & 1,85 & 6,74 & 1,49 & 7,24 & 1,53 & 6,93 & 1,40 \\
\hline
\end{tabular}




\begin{tabular}{ccccccccccc}
\hline Rep & \multicolumn{2}{c}{ T1 } & \multicolumn{2}{c}{ T2 } & \multicolumn{2}{c}{ T3 } & \multicolumn{2}{c}{ T4 } & \multicolumn{2}{c}{ T5 } \\
\hline 4 & 1,55 & 0,24 & 3,25 & 0,56 & 6,22 & 1,27 & 6,59 & 1,14 & 7,31 & 1,36 \\
5 & 4,91 & 0,88 & 10,27 & 1,98 & 10,62 & 2,11 & 1,94 & 0,33 & 10,53 & 2,08 \\
6 & 2,30 & 0,36 & 5,77 & 1,16 & 10,29 & 2,01 & 11,58 & 2,31 & 0,85 & 0,13 \\
7 & 5,08 & 0,79 & 7,19 & 1,32 & 6,44 & 1,13 & 0,30 & 0,04 & 4,30 & 0,77 \\
8 & 6,74 & 1,42 & 7,82 & 1,66 & 5,83 & 1,13 & 7,71 & 1,62 & 6,82 & 1,02 \\
9 & 3,23 & 0,54 & 5,46 & 1,13 & 5,90 & 1,08 & 11,42 & 2,29 & 8,92 & 1,90 \\
10 & 8,09 & 1,44 & 6,50 & 1,16 & 7,41 & 1,53 & 4,51 & 0,85 & 5,20 & 0,92 \\
\hline
\end{tabular}

Fuente: Autoría propia.

Después de 90 días de emergencia de las plántulas, se realizó el tercer corte. En este la planta fue removida, la parte aérea y el sistema radicular fueron medidos y pesados. Los resultados se describen en la Tabla 5.

Tabla 5 - Los resultados obtenidos relacionados con la producción de materia verde (MV), materia seca (MS), peso sistema radicular, medida sistema radicular y medida de la parte aérea por tratamiento $(T)$, por repetición (Rep), de la planta cosechada 90 días después de su emergencia

\begin{tabular}{ccccccccccc}
\hline T1 & $\mathbf{1}$ & $\mathbf{2}$ & $\mathbf{3}$ & $\mathbf{4}$ & $\mathbf{5}$ & $\mathbf{6}$ & $\mathbf{7}$ & $\mathbf{8}$ & $\mathbf{9}$ & $\mathbf{1 0}$ \\
\hline Aire/cm & 99,0 & 107,0 & 81,0 & 79,0 & 82,1 & 82,0 & 60,0 & 76,0 & 79,0 & 76,0 \\
Raíz/cm & 42,0 & 53,0 & 50,0 & 40,0 & 49,8 & 61,0 & 34,0 & 47,0 & 62,0 & 59,0 \\
Raíz/g & 17,58 & 17,95 & 10,1 & 6,34 & 16,55 & 24,69 & 6,49 & 26,97 & 24,25 & 14,63 \\
MV/g & 21,51 & 23,46 & 18,92 & 13,1 & 18,42 & 23,48 & 9,25 & 20,32 & 16,92 & 18,81 \\
MS/g & 5,31 & 5,81 & 4,26 & 2,38 & 4,07 & 5,81 & 1,39 & 4,25 & 3,89 & 3,53 \\
\hline T2 & $\mathbf{1}$ & $\mathbf{2}$ & $\mathbf{3}$ & $\mathbf{4}$ & $\mathbf{5}$ & $\mathbf{6}$ & $\mathbf{7}$ & $\mathbf{8}$ & $\mathbf{9}$ & $\mathbf{1 0}$ \\
\hline Aire/cm & 83,0 & 73,0 & 83,0 & 91,0 & 88,0 & 84,0 & 82,0 & 88,0 & 89,0 & 84,0 \\
Raíz/cm & 52,0 & 70,0 & 57,0 & 63,0 & 45,0 & 28,0 & 58,0 & 54,0 & 62,0 & 73,0 \\
Raíz/g & 21,84 & 19,13 & 21,85 & 24,15 & 19,03 & 9,05 & 26,23 & 23,02 & 27,81 & 25,12 \\
MV/g & 21,2 & 21,14 & 21,92 & 30,55 & 23,83 & 15,23 & 25,4 & 27,48 & 23,19 & 20,71 \\
MS/g & 5,03 & 3,97 & 5,22 & 7,72 & 5,76 & 3,07 & 5,24 & 6,77 & 5,30 & 4,73 \\
\hline T3 & $\mathbf{1}$ & $\mathbf{2}$ & $\mathbf{3}$ & $\mathbf{4}$ & $\mathbf{5}$ & $\mathbf{6}$ & $\mathbf{7}$ & $\mathbf{8}$ & $\mathbf{9}$ & $\mathbf{1 0}$ \\
\hline Aire/cm & 92,0 & 77,0 & 67,0 & 68,0 & 88,0 & 78,0 & 64,0 & 87,0 & 94,0 & 94,0 \\
Raíz/cm & 50,0 & 38,0 & 45,0 & 54,0 & 38,0 & 42,0 & 50,0 & 46,0 & 80,0 & 49,0 \\
Raíz/g & 27,23 & 13,97 & 14,94 & 18,09 & 13,34 & 25,53 & 18,48 & 14,53 & 13,22 & 25,1 \\
MV/g & 29,55 & 21,12 & 11,56 & 10,64 & 21,68 & 17,82 & 9,74 & 21,53 & 21,61 & 24,95 \\
MS/g & 7,38 & 4,98 & 2,52 & 2,50 & 4,41 & 4,99 & 2,12 & 4,58 & 4,11 & 6,19 \\
\hline
\end{tabular}




\begin{tabular}{ccccccccccc}
\hline T4 & $\mathbf{1}$ & $\mathbf{2}$ & $\mathbf{3}$ & $\mathbf{4}$ & $\mathbf{5}$ & $\mathbf{6}$ & $\mathbf{7}$ & $\mathbf{8}$ & $\mathbf{9}$ & $\mathbf{1 0}$ \\
\hline Aire/cm & 82,0 & 73,0 & 83,0 & 89,0 & 90,0 & 95,0 & 85,0 & 74,0 & 93,0 & 64,0 \\
Raíz/cm & 40,0 & 47,0 & 52,0 & 60,0 & 40,0 & 84,0 & 60,0 & 41,0 & 47,0 & 50,0 \\
Raíz/g & 23,34 & 8,07 & 22,62 & 19,27 & 21,14 & 42,36 & 26,84 & 10,34 & 16,36 & 8,72 \\
MV/g & 24,24 & 12,53 & 23,64 & 24,67 & 26,5 & 24,09 & 21,55 & 12,78 & 17,8 & 6,05 \\
MS/g & 5,45 & 1,82 & 5,77 & 5,40 & 6,39 & 5,73 & 4,51 & 2,75 & 4,42 & 1,33 \\
\hline T5 & $\mathbf{1}$ & $\mathbf{2}$ & $\mathbf{3}$ & $\mathbf{4}$ & $\mathbf{5}$ & $\mathbf{6}$ & $\mathbf{7}$ & $\mathbf{8}$ & $\mathbf{9}$ & $\mathbf{1 0}$ \\
\hline Aire/cm & 88,0 & 98,0 & 86,0 & 94,0 & 91,0 & 68,0 & 94,0 & 104,0 & 53,0 & 82,0 \\
Raíz/cm & 50,0 & 48,0 & 59,0 & 53,0 & 42,0 & 56,0 & 50,0 & 43,0 & 20,0 & 30,0 \\
Raíz/g & 25,17 & 50,7 & 23,26 & 20,49 & 23,76 & 12,44 & 20,28 & 25,25 & 3,72 & 4,16 \\
MV/g & 25,36 & 30,6 & 24,88 & 21,67 & 24 & 6,44 & 24,26 & 26,11 & 5,81 & 7,96 \\
MS/g & 5,29 & 7,33 & 4,76 & 4,58 & 5,54 & 1,41 & 5,76 & 6,01 & 0,96 & 1,41 \\
\hline Fu
\end{tabular}

Fuente: Autoría propia.

Para la evaluación de la MV y MS, se utilizó la prueba de Duncan con Alpha 0,1. Los resultados se describen en la Tabla 6 y 7, respectivamente.

Tabla 6 - Análisis estadístico de la MV de las plantas cosechadas 90 días después de la emergencia

\begin{tabular}{|c|c|c|}
\hline Duncan Grouping & Mean & TRAT \\
\hline$A$ & 25.269 & 5 \\
\hline$A$ & & \\
\hline A & 23.936 & 2 \\
\hline$A$ & & \\
\hline A & 23.213 & 4 \\
\hline$A$ & & \\
\hline A & 22.609 & 3 \\
\hline B & & \\
\hline B & 19.438 & 1 \\
\hline
\end{tabular}

Fuente: Autoría propia. 
Tabla 7-Análisis estadístico de la MS de las plantas cosechadas 90 días después de la emergencia

\begin{tabular}{|c|c|c|c|c|}
\hline \multicolumn{2}{|c|}{ Duncan Grouping } & \multirow{2}{*}{$\frac{\text { Mean }}{5.3233}$} & \multirow{2}{*}{$\frac{\mathbf{N}}{6}$} & \multirow{2}{*}{$\begin{array}{c}\text { TRAT } \\
5\end{array}$} \\
\hline & $A$ & & & \\
\hline & A & & & \\
\hline & A & 5.2133 & 6 & 2 \\
\hline & A & & & \\
\hline B & A & 4.8614 & 7 & 4 \\
\hline \multicolumn{5}{|c|}{ B } \\
\hline B & & 4.2650 & 6 & 3 \\
\hline \multicolumn{5}{|c|}{ B } \\
\hline B & & 3.9557 & 7 & 1 \\
\hline
\end{tabular}

Fuente: Autoría propia.

De acuerdo con la prueba realizada, el uso de cenizas de vinaza como fertilizante, independientemente de la cantidad, se mostró efectivo, pues la producción de MV y MS del testigo, fue inferior, cuando comparada a los tratamientos. Sin embargo, el tratamiento 5 se mostró más eficaz, pero sin diferencia estadística del tratamiento 2, es decir, el uso del tratamiento 2 es más indicado por ser económicamente viable con relación al tratamiento 5.

Bonfim-Silva et al., (2011b) utilizando ceniza vegetal aumentaron la producción de materia seca de la parte aérea de plantas de crotalaria juncea en el 89,38\% y Bonfim-Silva et al. (2011c) aplicando ceniza vegetal en plantas de arúgula obtuvieron un aumento en la producción de materia verde de la parte aérea del 89,14\%. La ceniza vegetal proporciona reducción en el tiempo para la forrajera alcanzar la altura de $50 \mathrm{~cm}$ y proporciona un aumento en la altura de las plantas, convirtiéndose en una alternativa de costo bajo para manejar los pastizales (Bonfim-Silva et al., 2011a).

Verificó en este trabajo, aumento en la producción de masa seca del pasto marandu. Estos resultados corroboran con los obtenidos por Santos (2012) que trabajó con este mismo cultivar de Urochloa brizantha y con Paterson et al. (2004) trabajando con plantas de cebada que evidenciaron el efecto positivo de la ceniza vegetal en la producción de estos cultivos.

El aumento en la producción del número de hojas en pasto marandu ocurrió de forma significativa siendo constatado por Bonfim-Silva et al. 
(2011a) cuando la aplicación de ceniza vegetal. Se observó un aumento en el número de hojas en el primer y segundo crecimiento de este cultivar de Urochloa brizantha. El aumento en el número de hojas posibilitó la restauración foliar después del pastoreo o corte lo que ayudó al mantenimiento y la longevidad de la gramínea (CUNHA et al., 2011).

La presencia de perfiles en la gramínea aumenta su perennidad y su establecimiento, asegurando mayor resistencia contra la invasión de malas hierbas, plagas y enfermedades, determinando la producción del forraje (PEDREIRA; MELO; OTANI, 2001). La existencia del perfilado es factor determinante en la producción y va a depender del estadio vegetal de la planta (SILSBURY, 1966). También el aumento en el peso de la planta es determinado por la presencia de los perfiles existentes (SILVA; PEDREIRA, 1997).

La ceniza vegetal posee los macro y micronutrientes necesarios para las plantas y que pueden estar presentes en mayor o menor concentración (VAMVUKA; ZÓGRAFOS, 2004). Pedreira, Melo e Otani (2004) confirman que el sistema radicular es el primero que sufre los impactos de un manejo inadecuado cuando aliado a la baja fertilidad del suelo y que las cenizas juegan un papel importante en el desarrollo de las raíces de las plantas.

\section{CONCLUSIÓN}

Se concluye que el uso de cenizas de vinaza es una alternativa viable cuando se utiliza en la proporción de $100 \mathrm{~kg} / \mathrm{ha}$ pues elevó en promedio el 18,5\% la producción de MV y el 24\% la producción de MS de la forrajera Urochloa brizantha cv. Marandu.

\section{REFERENCIAS}

BONFIM-SILVA, E. M.; SILVA, T. J. A.; CABRAL, C. E. A.; VALADARES, E. M.; GOLDONI, G.. Características morfológicas e estruturais de capim marandu adubado com cinza vegetal em Latossolo Vermelho do Cerrado. Enciclopédia Biosfera, Jandaia, v. 7, n. 12, p. 1-9, 2011 a.

BONFIM-SILVA, E. M.; GUIMARÃES, S. L. C.; SILVA, T. J. A.; NEVES, L. C. R.; SILVA, T. J. A. Desenvolvimento e produção de Crotalária juncea adubada com cinza vegetal. Enciclopédia Biosfera, Jandaia, v. 7, n. 13, p. 371-9, 2011 b. 
BONFIM-SILVA, E. M.; SILVA, T. J. A.; SANTOS, C. C.; CABRAL, C. E. A.; SANTOS, I. B.. Características produtivas e eficiência no uso de água em rúcula adubada com cinza vegetal. Enciclopédia Biosfera, Jandaia, v. 7, n. 13, p. 178-86, 2011c.

COSTA, K. A. P.; ARAÚJO, J. L.; FAQUIN, V.; OLIVEIRA, I. P.; FIGUEIREDO, F. C.; GOMES, K. W. Extração de macronutrientes pela fitomassa do capim-xaraés em função de doses de nitrogênio e potássio. Ciência Rural, Santa Maria, v. 38, n. 4, p. 1162-6, 2008.

CUNHA, F. F.; RAMOS, M. M.; ALENCAR, C. A. B.; OLIVEIRA, R. A.; ARAUJO, R. A. S.; CECON, P. R.; MARTINS, C. E.; COSER, A. C. Número de folhas do capim-xaraés em diferentes manejos e doses de adubação, intervalos de desfolha e estações Anuais. Bioscience Journal, Uberlândia, v. 27, n. 2, p. 271-82, 2011.

DECHEN, A. R.; NACHTIGALL, G. R. Elementos requeridos à nutrição de plantas. In: NOVAES, R. F.; ALVAREZ, V. V. H.; BARROS, N. F.; FONTES, R. L. F.; CANTARUTTI, B.; NEVES, J. C. L. Fertilidade do solo. Viçosa: Sociedade Brasileira de Ciência do Solo, 2007. p. 676-80.

FERREIRA, E. P. B.; FAGERIA, N. K.; DIDONET, A. D. Chemical properties of an Oxisol under organic management as influenced by application of sugarcane bagasse ash. Revista Ciência Agronômica, Fortaleza, v. 43, p. 228-36, 2012.

MALAVOLTA, E. Manual de calagem e adubação das principais culturas. São Paulo: Ceres, 1987. 496 p.

MONTEIRO, F. A.; RAMOS, A K. B.; CARVALHO, D. D.; ABREU, J. B. R.; DAIUB, J. A. S.; SILVA, J. E. P.; NATALE, W. Cultivo de Brachiaria brizantha stapf. cv. marandu em solução nutritiva com omissões de macronutrientes. Scientia Agricola, São Paulo, v. 52, n. 1, p. 135-41, 1995.

OLIVEIRA, P. P. A.; TRIVELIN, P. C. O.; OLIVEIRA, W. S.; CORSI, M.. Fertilização com N e S na recuperação de pastagens de Brachiaria brizantha cv. Marandu em Neossolo quartzarênico. Revista Brasileira de Zootecnia, Viçosa, v. 34, n. 4, p. 1121-9, 2005.

PATTERSON, S. J.; ACHARYA, S. N.; THOMAS, J. E.; BERTSCHI, A.B.; ROTHWELL, R. L. Barley biomass and grain yield and canola seed yield response to land application of wood ash. Agronomy Journal, v. 96, n.4, p. 971-7, 2004.

PEDREIRA, C. G. S.; MELLO, A. C. L.; OTANI, L. O processo de produção de forragem em pastagens. In: REUNIÃO ANUAL DA SOCIEDADE BRASILEIRA DE ZOOTECNIA, 38., Piracicaba, 2001. Anais [...]. Piracicaba: Sociedade Brasileira de Zootecnia, 2001. 
PEDREIRA, C. G. S.; MOURA, J. C.; FARIA, V. P. Fertilidade do solo para pastagens produtivas. In: SIMPÓSIO SOBRE MANEJO DA PASTAGEM, Piracicaba, 2004. Anais [...]. Piracicaba: FEALQ, 2004.

RAIJ, B. V. Fertilidade do solo e adubação. São Paulo: Ceres, 1991. 343 p.

SANTOS, C. C. Cinza vegetal como corretivo e fertilizante para Marandu e Xaraés. 2012. 127f. Dissertação (Mestrado em Engenharia Agrícola) - Universidade Federal de Mato Grosso, Rondonópolis, MT, 2012.

SILVA, S. C.; PEDREIRA, C. G. S. Princípios de ecologia aplicados ao manejo da pastagem. In: ECOSSISTEMA DE PASTAGENS, 3., 1997, Jaboticabal. Anais [...]. Jaboticabal: UNESP, 1997. p. 1-62.

SILSBURY, J. H. Interrelations in the growth and development of Lolium. II. Tiller number and dry weight at low density. Australian Journal of Agricultural Research, Clayton South, v. 17, n. 6, p. 841-7, 1966.

VAMVUKA, D.; ZÓGRAFOS, S. Predicting the behaviour of ash from agricultural waste during combustion. Fuel, Crete, v. 83, p. 2051-7, 2004.

VILELA, L.; SOUZA, D.M.G.; MARTHA JÚNIOR, G.B. Calagem. In: MARTHA JÚNIOR, G.B.; VILELA, L.; SOUSA, D.M.G. Cerrado, uso eficiente de corretivos e fertilizantes em pastagens. Planaltina, 2007. p. 93-106. 